\title{
Rational Approach to Chatter in Venovenous Extracorporeal Membrane Oxygenation to Limit Fluid Administration: An Algorithmic Description
}

\author{
Vinay Krishnamurthy ${ }^{1}$, Sunil Karanth ${ }^{2}$ \\ Keywords: Chatter, Drainage insufficiency, Extracorporeal membrane oxygenation. \\ Indian Journal of Critical Care Medicine (2022): 10.5005/jp-journals-10071-24113
}

\section{INTRODUCTION}

Extracorporeal membrane oxygenation (ECMO) is considered an effective rescue therapy for severe acute lung or cardiac disease. ${ }^{1,2}$ It is important to understand and troubleshoot basic circuit-related complications which may uniquely impact outcome. One such challenge being chatter, which is considered sine qua non for fluid-deficient status, hence leading to irrational fluid administration. Results of the fluids and catheters treatment trial (FACTT) in 2007 demonstrated that conservative fluid strategy for acute respiratory distress syndrome (ARDS) reduces net fluid balance and improves outcomes in oxygenation and ventilator-free day, when compared to the liberal strategy. ${ }^{3}$ A study done by Schmidt et al. showed that early positive fluid balance at day 3 is a robust independent predictor of 90-day mortality during ECMO, regardless of primary diagnosis, acute kidney injury, or renal replacement therapy. ${ }^{4}$ Hence, the fluid should be administered with caution, only after excluding all other causes for drainage insufficiency.

\section{Pathophysiology}

Modern centrifugal pumps are preload-dependent, and blood flow through the drainage cannula is governed by Hagen-Poisuelle formula. When the drainage cannula suction (in-turn a function of rotations of the pump) is set higher than the venous load, it leads to collapse of non-rigid vena cava, obstructing the drainage ports causing interrupted blood flow and drainage chatter. As the blood reaccumulates, transient relieving of the obstruction occurs. This vicious cycle of variable blood flow leads to chatter. Chatter can be described as rhythmic pulsations of the ECMO tubing due to erratic nonlaminar blood flow as shown in Figure 1. The main drivers of drainage insufficiency are insufficient venous capacitance and/or excessive negative pressure.

Causes that lead to insufficient capacitance are hypovolemia, vasodilation, Valsalva maneuver, inflow obstruction, and small drainage cannula. Increased intra-abdominal pressures affect the capacitance of the inferior venacava (IVC), whereas increased intrathoracic pressures like pneumothorax, tamponade, Valsalva may affect drainage to superior venacava (SVC) and/or right atrium. Excess negative drainage pressure can result from high pump speed relative to inflow resistance and/or blood volume. This effect is exacerbated when there is a concomitant insufficient venous return.

\footnotetext{
1,2Department of Critical Care Medicine, Manipal Hospital, Bengaluru, Karnataka, India
}

Corresponding Author: Vinay Krishnamurthy, Department of Critical Care Medicine, Manipal Hospital, Bengaluru, Karnataka, India, Phone: +91 9738515164, e-mail: vinayktcfc@gmail.com

How to cite this article: Krishnamurthy V, Karanth S. Rational Approach to Chatter in Venovenous Extracorporeal Membrane Oxygenation to Limit Fluid Administration: An Algorithmic Description. Indian J Crit Care Med 2022;26(2):244-245.

Source of support: Nil

Conflict of interest: None

Chatter occurring soon after cannulation also called "postcannulation syndrome" may be due to vasodilation as a part of systemic inflammatory response syndrome(SIRS) response upon exposure of blood to the extracorporeal circuit. Other causes being undersized cannula, malposition, and vascular injury. ${ }^{5}$ Later in the course, drainage insufficiency can be due to increased cardiac output states, sepsis, agitation due to inadequate sedation, and volume depletion.

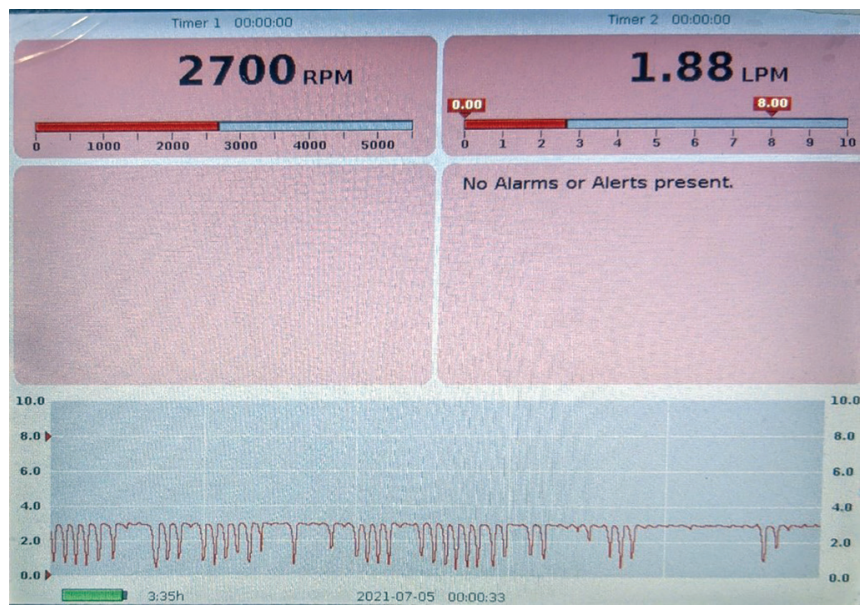

Fig. 1: Trend of blood flows on ECMO monitor showing chatter

(-) The Author(s). 2022 Open Access This article is distributed under the terms of the Creative Commons Attribution 4.0 International License (https://creativecommons. org/licenses/by-nc/4.0/), which permits unrestricted use, distribution, and non-commercial reproduction in any medium, provided you give appropriate credit to the original author(s) and the source, provide a link to the Creative Commons license, and indicate if changes were made. The Creative Commons Public Domain Dedication waiver (http://creativecommons.org/publicdomain/zero/1.0/) applies to the data made available in this article, unless otherwise stated. 
Clinicians should first confirm that chatter is occurring in the prepump drainage tubing. Isolated postpump chatter may be due to blood flow at higher velocities within the tubing. If available, monitoring pressure in the venous drainage cannula (less than $-100 \mathrm{~mm} \mathrm{Hg}$ ) may help in the early prediction of chatter. ${ }^{6}$ A crude indicator of drainage insufficiency is failure to appreciably increase the blood flow in response to increasing the pump speed.

The most important complication of chatter is progressive hypoxemia due to decreased flow through the ECMO circuit. Due to strain forces and excess negative pressure, there can be an injury to cellular components which can lead to hemolysis and cavitation. If left untreated, this may progress to complete flow cessation due to "suck down." Greater incidence of circuit thrombosis and oxygenator dysfunction is also encountered frequently. ${ }^{6}$

\section{Management of Chatter}

Let us confirm the chatter is of the drainage cannula. Timeline of chatter during the course of ECMO may help to narrow the diagnosis. Stepwise approach should be followed as shown in Flowchart 1. The first step is to reduce the pump speed so as to achieve a stable blood flow, if the patient is adequately compensated; if not, attempts should be made gradually to set a speed below a level where a further increase in rotations will not lead to an increase in blood flow. It should be ensured that patient is not agitated, restless or coughing, and adequately sedated. The next step is to inspect the circuit from end to end to ensure that there is no obstruction and to identify occult bleeding sites and to check for cannula position, especially femoral drainage cannula and evaluate for causes of increased intra-abdominal pressure (e.g., intra-abdominal hypertension) and increased intrathoracic pressures (e.g., pneumothorax, pericardial tamponade, etc.) as shown in Figure 2. If the chatter continues, then it is prudent to give a volume challenge by auto-infusion through Trendelenburg position. If the chatter

Flowchart 1: Algorithm for identification of etiology of chatter

\begin{tabular}{|c|c|}
\hline \multicolumn{2}{|c|}{ Correct identification of drainage cannula chatter } \\
\hline & $\begin{array}{l}\text { Tiiming and } \\
\text { clinical scenario }\end{array}$ \\
\hline \multicolumn{2}{|c|}{ Reduce the pump speed until stable blood flow is obtained } \\
\hline & If persists \\
\hline \multicolumn{2}{|c|}{ Ensure patient is not agitated/coughing/restless-adequate sedation } \\
\hline & If persists \\
\hline \multicolumn{2}{|c|}{$\begin{array}{l}\text { Inspection of tubing for obstruction, look for occult bleeding } \\
\text { sites look for malpositioning of cannula/clots }\end{array}$} \\
\hline & If persists \\
\hline \multicolumn{2}{|c|}{$\begin{array}{l}\text { To look for causes of increased intrathoracic and } \\
\text { intrabdominal pressures }\end{array}$} \\
\hline & If persists \\
\hline \multicolumn{2}{|c|}{$\begin{array}{l}\text { Assess for fluid responsiveness by giving trendelenburg position, } \\
\text { if responsive state with fluid resuscitaion with small fluid boluses }\end{array}$} \\
\hline & If persists \\
\hline $\begin{array}{r}\text { Consider additional draina } \\
\text { desired ECMO }\end{array}$ & $\begin{array}{l}\text { age cannula to meet the } \\
\text { O blood flow }\end{array}$ \\
\hline
\end{tabular}

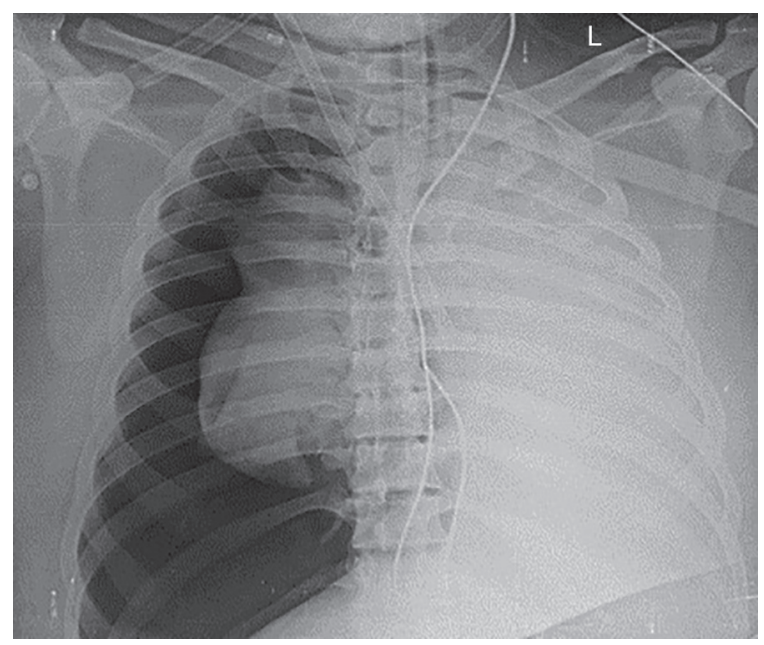

Fig. 2: Radiograph depicting right pneumothorax in a patient on venovenous (VV)-ECMO

subsides, it indicates the scope for fluid administration in small aliquots. In spite of all these measures, if chatter persists, then the possibility of additional drainage cannula needs to be considered. Hence, we need to select adequately sized drainage cannula, e.g., $23 \mathrm{Fr}$ or above, to provide adequate drainage at lower drainage pressures. Appropriate titration of flow depending on the recovery of the native lung function is also equally important.

\section{Conclusion}

Comprehensive assessment of the clinical scenario and timeline of chatter during the course of ECMO is important to identify the probable aetiology. A thoughtful physiological algorithm-based approach may help limit harmful outcomes associated with irrational fluid administration in ARDS.

\section{OrCID}

Vinay Krishnamurthy @ https://orcid.org/0000-0002-8936-0605 Sunil Karanth @ https://orcid.org/0000-0003-3597-4473

\section{References}

1. Brodie D, Bacchetta M. Extracorporeal membrane oxygenation for ARDS in adults. N Engl J Med 2011;365(20):1905-1914. DOI: 10.1056/ NEJMct1103720.

2. Sayer GT, Baker JN, Parks KA. Heart rescue: the role of mechanical circulatory support in the management of severe refractory cardiogenic shock. Curr Opin Crit Care 2012;18(5):409-416. DOI: 10.1097/MCC.0b013e328357f1e6.

3. Wiedemann HP, Wheeler AP, Bernard GR, Thompson BT, Hayden D, deBoisblanc $B$, et al. Comparison of two fluid-management strategies in acute lung injury. N Engl J Med 2006;354(24):2564-2575. DOI: 10.1056/NEJMoa062200.

4. Schmidt M, Bailey M, Kelly J, Hodgson C, Cooper DJ, Scheinkestel C, et al. Impact of fluid balance on outcome of adult patients treated with extracorporeal membrane oxygenation. Intensive Care Med 2014;40(9):1256-1266. DOI: 10.1007/s00134-014-3360-2.

5. Davies MG, Hagen PO. Systemic inflammatory response syndrome. Br J Surg 1997;84(7):920-935. DOI: 10.1002/bjs.1800840707.

6. Faghih MM, Sharp MK. Modeling and prediction of flow-induced hemolysis: a review. Biomech Model Mechanobiol 2019;18(4):845881. DOI: 10.1007/s10237-019-01137-1. 